\title{
Germs, genomics and global public health
}

\author{
How can advances in genomic sciences be integrated into public \\ health in the developing world to deal with infectious diseases?
}

\author{
T. Pang
}

Received: 28 June 2009/Accepted: 23 October 2009/Published online: 5 November 2009

(C) Springer Science+Business Media B.V. 2009

\begin{abstract}
Scientific and technological advances derived from the genomics revolution have a central role to play in dealing with continuing infectious disease threats in the developing world caused by emerging and re-emerging pathogens. These techniques, coupled with increasing knowledge of host-pathogen interactions, can assist in the early identification and containment of outbreaks as well as in the development of preventive vaccination and therapeutic interventions, including the urgent need for new antibiotics. However, the effective application of genomics technologies faces key barriers and challenges which occur at three stages: from the research to the products, from the products to individual patients, and, finally, from patients to entire populations. There needs to be an emphasis on research in areas of greatest need, in facilitating the translation of research into interventions and, finally, the effective delivery of such interventions to those in greatest need. Ultimate success will depend on bringing together science, society and policy to develop effective public health implementation strategies to provide health security and health equity for all peoples.
\end{abstract}

Keywords Genomics - Infectious diseases ·

Developing countries Public health

Abbreviations
ANDI $\quad \begin{aligned} & \text { African network for drugs and diagnostics } \\ & \text { innovation }\end{aligned}$
EPI $\quad$ Expanded programme on immunization

T. Pang $(\bowtie)$

Research Policy \& Cooperation, World Health Organization, Avenue Appia, 1211 Geneva 27, Switzerland

e-mail: pangt@who.int
ICH-GCP International conference on harmonizationgood clinical practice

MDR Multi drug resistant

MRSA Methicillin-resistant Staphylococcus aureus

NIH National Institutes of Health

WHO World Health Organization

XDR Extensively drug resistant

\section{Introduction}

Unprecedented advances have been made in the advanced genomic sciences with regards to new knowledge and powerful technologies which relate to infectious diseases. However, a major challenge is to ensure that these advances have an impact on public health improvement and policies, especially in the developing world. The present article will identify the major infectious disease threats, the relevant technologies, the barriers and hurdles which need to be overcome, and the policy responses which need to be instituted to successfully meet the challenges.

\section{Infectious disease threats}

Developing countries continue to struggle with emerging and re-emerging infectious diseases. Recent outbreaks of cholera (in Zimbabwe), meningitis and Ebola (in Africa), chikungunya (in Southeast Asia, India, Sri Lanka, Kenya), foot-and-mouth diseases (in China) and dengue (in Bolivia, Southeast Asia) underscores the continuing threat in many parts of the world. Avian influenza remains a major public health threat and, based on studies of the 1918 global flu pandemic, it has been estimated that a recurrence of such a pandemic will kill between 50 and 80 million people 
worldwide, $95 \%$ of them in the developing world (Murray et al. 2006), at an estimated economic cost of $\$ 3$ trillion. In addition, the threat of new pathogens emerging was illustrated by the appearance of a new arenavirus in South Africa in 2008 which killed four out of five people it infected (Keeton 2008). In addition, there are continued concerns with the possibility and risk of pathogens crossing the animal to human transmission barrier (Normile 2009).

Antibiotic resistance constitutes another major public health threat (Taubes 2008) and includes MDR- (multi-drug resistant) and XDR-TB (extensively-resistant tuberculosis), MRSA (methicillin-resistant Staphylococcus aureus), vancomycin-resistant Enterococcus and MDR Salmonella typhi. In addition, there are concerns with the recent appearance of resistance to artemisinin-combined therapy for malaria on the Thai-Cambodia border and emergence of resistance among influenza viruses to the antiviral agent oseltamivir (tamiflu). It has been estimated that by $2009,98 \%$ of circulating influenza $\mathrm{A} / \mathrm{H} 1 \mathrm{~N} 1$ virus strains in North America have become resistant to oseltamivir (Layne et al. 2009) and there are worrying reports of resistance developing among the much more virulent H5N1 strain of influenza. In a recent analysis of emerging and re-emerging infectious disease threats globally, Jones et al. (2008) showed that approximately $60 \%$ of these threats came from zoonoses or wildlife origins (e.g. wild birds in the case of avian influenza, bats in the case of Nipah virus, etc.), $20 \%$ were vector borne agents and $20 \%$ were due to antibiotic-resistant bacteria. "Hot spots" of emergence of these threats were almost exclusively located in the developing world, i.e. in tropical Asia, central America and tropical Africa. Importantly, and ironically, resources for surveillance and investigation of these threats are focused in areas where the next emerging pathogen is least likely to come from, i.e. in the developed world.

Another important threat exists in the context of the current global financial crisis (Horton 2009) and the reality that the health budget is often the first to be cut in developing countries in times of scarce resources. It is also likely that overseas development aid, which is relied upon by many low-income countries for a significant portion of their health budgets, may also be reduced in the coming years. This may further weaken many health care delivery systems in low and middle-income countries which are already in a weak and fragile state, thus affecting their ability to effectively respond to infectious disease threats.

\section{Technologies derived from genomics}

The genomics and post-genomics era has been accompanied by an impressive array of new and powerful technologies which have a direct impact on the diagnosis, control and prevention of infectious diseases. An analysis of the top ten biotechnologies for improving health in developing countries identified diagnostics, vaccines and drug and vaccine delivery as the top three technologies (Daar et al. 2002). In broader terms, the list of key technologies include the following:

1. The rapid identification of pathogens-this is the cornerstone of surveillance and detection of new or reemerging pathogens and relies on the rapid sequencing of pathogen sequences followed by comprehensive, comparative analysis with genomic sequences present in openly accessible databases.

2. Rapid, cheap, easy to use, point of care diagnosticsideally, these tests should be able to detect multiple pathogens and be usable in least developed settings. Better tests for TB and malaria, for example, can have a significant impact on the burden of disease in affected areas (Mabey et al. 2004).

3. Better, more effective, easily deployable vaccinesbeyond the traditional vaccines used in the EPI (Expanded Programme on Immunization) there is also a need to better utilize existing vaccines (e.g. against typhoid, pneumonia) and develop more effective vaccines in the future (e.g. against TB, malaria, HIV/ AIDS).

4. Genomics in drug discovery-pathogen genome sequences, combined with other approaches such as access to chemical libraries and molecular-target high throughput screening, are the starting points of many drug discovery and vaccine development initiatives directed at neglected diseases affecting developing countries e.g. the African Network for Drugs and Diagnostics Innovation (ANDI) and Aeras, which aims to develop new, safe and effective vaccine regimens to prevent tuberculosis in children, adolescents and adults.

5. Applications to vector control-the recent availability of complete genome sequences for some major disease vectors (e.g. Aedes spp., Anopheles spp.) will see some important future applications for the control of these disease-transmitting mosquitoes, including strategies to reduce or eliminate their competence as vectors of disease (Povelones et al. 2009).

6. Monitoring resistance (viral, bacterial, protozoal)molecular markers for development of resistance to anti-microbial agents, rapidly deployed together with diagnostic tests, is critical for surveillance and appropriate therapeutic responses.

7. Translating new knowledge of host-pathogen interactions into better interventions - there is an increasingly important body of knowledge which links infections with the immune and inflammatory responses, including 
the genetic susceptibility to infectious diseases. Advances in this field have important implications for the development of therapeutics and diagnostics in the future. Pharmacogenomics and personalized medicine, for example, promises to improve drug safety and efficacy (McGuire et al. 2007; Katsanis et al. 2008). A recent study by Nijnik and Hancock (2009) described the antimicrobial and immunomodulatory activities of host-defence peptides and their potential applications for tackling antibiotic-resistant infections.

With regard to future technologies, it is also useful to consider areas where technology development is needed for dealing with infectious disease threats. A "window" into what might constitute these priority research areas can be gleaned from the recently announced US NIH Challenge Grants in Health and Science Research (NIH 2009) which highlighted the following as some of the priority areas:

1. Development of biomedical technologies and systems, including the provision of "immediate diagnostic information for multiple conditions at the point-ofcare";

2. New computational and statistical methods for the analysis of large data sets from next-generation sequencing technologies;

3. Theranostics - the combined delivery of diagnostic and therapeutic agents;

4. Develop diagnostics and drugs for multiple- and extensively drug-resistant tuberculosis;

5. Develop drugs for neglected tropical diseases, with a special emphasis on malaria;

6. Novel approaches to improve immunogenicity of vaccines against small molecules.

It is also important to emphasize that genomics-derived technologies alone are not sufficient to deal with infectious disease threats. A Foresight Report from the United Kingdom has identified that, in addition to genomics and postgenomic approaches for rapid characterization of pathogens, it is also important to consider innovative IT (information technology) applications to capture, analyse and model data (e.g. the use of hand held devices); non invasive mass screening of people, animals and plants; and chip-based portable diagnostic devices (King et al. 2006).

\section{Barriers and challenges}

The effective application of the technologies mentioned above faces some key barriers and challenges which occur at three stages: from the research to the products, from the products to individual patients, and, finally, from patients to entire populations.
Barrier 1-The R\&D barrier: from publications to products

The central questions here is: "are we doing the right science to get the appropriate knowledge and interventions with regards to both the areas and the types of research?

It has been estimated that approximately $\$ 160$ billion was invested in global health research \& development globally, compared to $\$ 85$ billion in 1998 and $\$ 30$ billion in 1986 (Burke and Matlin 2008). Despite this influx of funds, gaps and imbalances continue to exist in the research process. The existence of the "10/90 gap", for example, is well known where only $10 \%$ of global health research funding is spent on $90 \%$ of the global disease burden. There also exists a well-known market failure in producing drugs for neglected tropical diseases in the developing world — of 1,556 new drugs developed between 1975-2004, only 21 (1.3\%) were for these diseases (Chirac and Torreele 2006). In terms of support for various research areas, a recent report analyzing funding patterns among more than 100 donors found that $80 \%$ of the funding were allocated to HIV/AIDS, TB and malaria (Moran et al. 2009). In another analysis of the types of research funded by the major UK agencies, it was found that an average of only $1 \%$ of funding was allocated to health services research (Rothwell 2006) with the bulk of the funding going to biomedical, clinical and epidemiological research.

More tellingly, most of the funding for health research is focused on the development of new technologies, rather than making use of existing interventions-in one analysis, $97 \%$ of grants were for the development of these new technologies which was estimated to be able to reduce child mortality by $22 \%$ (Leroy et al. 2007). However, if, instead, research were focused on making better use of existing technologies, the reduction in mortality can be much higher at $66 \%$. In the future, a lot more attention needs to be paid to this field of implementation research.

\section{Barrier 2-the translation barrier: from products to patients}

In the context of this barrier the key question is "do we have the most efficient processes to ensure that effective products benefits the patients?" In spite of the large amounts of resources going into global health $R \& D$, the number of new drugs in the pipeline is actually decreasing as exemplified by the lower number of new antibiotics coming on the market. Clinical trials, the cornerstone of getting drugs and other interventions to patients, are facing major problems with regards to high costs, difficulties in recruitment of trial participants, more regulations and concerns about transparency and accountability. These 
concerns include unethical behavior on the part of pharmaceutical companies, publication bias, and the nonreporting of adverse events or negative results. Importantly, the developing countries will be affected as it has been estimated that nearly $20 \%$ of clinical trials globally now take place in the developing world, up from in $9 \%$ in 2003 (Normile 2008). Most of the increases are occurring in Russia, India, China and Brazil. The main reasons for increasing interest in conducting trials in the developing world relate to lower costs, accelerated recruitment and perceived ease of overcoming ethical and regulatory barriers. There is also some concern about the over-regulation of clinical trials, specifically the requirements to adhere to the ICH-GCP guidelines which places too much emphasis on the process rather than on the benefits to the patients (White 2006; Farrar 2007). These bureaucratic requirements also place clinical trials beyond the reach of most developing country investigators and disempowers them from truly participating in the research and sharing in the benefits.

Inefficiencies have also been noted in the diagnostics field and an important question in this regard is "what is the human and economic costs of bad diagnostics?" Many developing countries lack stringent evaluation of diagnostic tests and, in the case of dengue diagnostics, for example, the claims of manufacturers were found to be well short of acceptable standards (Blacksell et al. 2006). In a more comprehensive analysis of diagnostic evaluations reported in journals from 1978-1993, it was found that less than half of the studies fulfilled more than three of the seven methodological standards for diagnostics (Peeling et al. 2006).

\section{Barrier 3-the implementation barrier: from patients to people}

In some ways this is the most challenging of the three barriers and focuses on the question "do we have the needed delivery systems to get interventions to those in greatest need?" Health systems failures and lack of access to the most basic interventions are at the heart of the problem. For example, Jones et al. (2003) found that less than $50 \%$ of children in the developing world were receiving basic, proven, effective and cheap interventions such as vitamin A, tetanus immunization and insecticide-treated bednets. Tragically, the gap in coverage for four interventions (family planning, maternal and neonatal care, immunization, and treatment of sick children) was larger for the poorer segments of the population living in these countries (WHO 2008). There are many reasons for these failures of delivery but foremost among them are the shortage of health workers, lack of reliable health information, insufficient financing and poor infrastructure. In relation to health workers, for example, Africa, which bears $25 \%$ of the global burden of disease only has $2-3 \%$ of the global health work force (WHO 2006). In addition, in many low-income countries the recent deluge of external aid delivered through multiple global health initiatives has created additional problems and strains on already weak and fragile health systems. There is evidence of fragmentation and lack of coordination between these initiatives and they tend to be "top down" and donor-driven, with a focus on short-term results around "big" diseases, thus creating vertical "silos", rather than overall health system strengthening. In Haiti, for example, a focus on HIV/AIDS testing and treatment resulted in the neglect of testing for syphilis with the result that children born to HIV-positive mothers were avoiding HIV/AIDS but dying of syphilis (Peeling et al. 2004). With many of these initiatives there is also a lack of evaluation of the impact of their activities and limited accountability mechanisms, thus bringing into question the longer term sustainability of the activities.

In the context of this third barrier, another important challenge is the weak linkages which exist between research and policy development. Policy makers and researchers have conflicting interests and often do not trust each other (WHO 2004). Importantly, researchers need to present science in a way which is understandable to policy makers and able to answer the three questions they are most concerned with: "can it work, will it work, is it worth it?" As stated previously in a quote attributed to Sir Michael Marmot, the reality is that "scientific findings do not fall on blank minds that get made up as a result. Science engages with busy minds that have strong views about how things are and ought to be" and it is therefore important that researchers and policy makers work together, perhaps through "knowledge brokerage" mechanisms to more effectively use scientific evidence in health policy development. In the context of application of technological advances derived from genomics this is particularly important as the science is often complex and the benefits not immediately obvious to the policy- and decisionmakers.

\section{Conclusions}

Genomics has made great strides in the past decade and its importance for the diagnosis, treatment, prevention and control of infectious diseases remains paramount. However, and in order to have an impact on global public health, the unprecedented scientific advances must be complemented with efforts to apply resulting knowledge and technologies to mitigate threats to health in the developing world. Barriers between research and public health application must be addressed and the mantra of "sequencing, sequencing, 
sequencing" should be counterbalanced by a call for "analysis, analysis, analysis", to which one can arguably add a plea for "application, application, application".

Effective application, in turn depends on putting in place the appropriate policy responses which should include better alignment, harmonization and coordination of genomics research so it addresses neglected areas. This should be accompanied by the development of appropriate capacity in developing countries through equitable partnerships, open and rapid sharing of information, developing inter-sectoral links and the facilitation of translation of research to benefit patients. Finally, we need to develop a more holistic, systems perspective to strengthen health systems through better links of research to policy to achieve scale up, impact and, ultimately, better health outcomes and equity.

\section{References}

Blacksell SD, Newton PN, Bell D et al (2006) The comparative accuracy of 8 commercial rapid immunochromatographic assays for the diagnosis of acute dengue virus infection. Clin Infect Dis 42:1127-1134

Burke MA, Matlin S (eds) (2008) Monitoring Financial Flows for Health Research 2008-Prioritizing research for health equity. Global Forum for Health Research, Geneva

Chirac P, Torreele E (2006) Global framework on essential health R\&D. Lancet 367:1560-1561

Daar AS, Thorsteinsdottir H, Martin DK et al (2002) Top ten biotechnologies for improving health in developing countries. Nat Genet 32:229-232

Farrar J (2007) Global health science: a threat and an opportunity for collaborative clinical science. Nat Immunol 8:1277-1279

Horton R (2009) The global financial crisis: an acute threat to health. Lancet 373:355-356

Jones G, Steketee RW, Black RE et al (2003) How many child deaths can we prevent this year? Lancet 362:65-71

Jones KE, Patel NG, Levy MA et al (2008) Global trends in emerging infectious diseases. Nature 451:990-993

Katsanis SH, Javitt G, Hudson K et al (2008) A case study of personalized medicine. Science 320:53-54

Keeton K (2008) South African doctors move quickly to contain new virus. Bull WHO 86:912-913
King DA, Peckham C, Waage JK et al (2006) Infectious diseases: preparing for the future. Science 313:1392-1393

Layne SP, Monto AS, Taubenberger JK (2009) Pandemic influenza: an inconvenient mutation. Science 323:1560-1561

Leroy JL, Habicht JP, Pelto G et al (2007) Current priorities in health research funding and lack of impact on the number of child deaths per year. Am J Public Health 97:219-223

Mabey D, Peeling RW, Ustianowski A et al (2004) Diagnostics for the developing world. Nat Revs Microbiol 2:231-240

McGuire AL, Cho MK, McGuire SE et al (2007) The future of personal genomics. Science 317:1687

Moran M, Guzman J, Ropars AL et al (2009) Neglected disease research and development: how much are we really spending? PLoS Med 6:e1000030

Murray CJL, Lopez AD, Chin B et al (2006) Estimation of potential global pandemic influenza mortality on the basis of vital registry data from the 1918-20 pandemic: a quantitative analysis. Lancet 368:2211-2218

NIH. National Institutes of Health. NIH Challenge Grants in Health and Science Research. http://grants.nih.gov/grants/funding/ challenge_award/ (Accessed on April 9, 2009)

Nijnik A, Hancock REW (2009) Host defence peptides: antimicrobial and immunomodulatory activity and potential applications for tackling antibiotic-resistant infections. Emerg Health Threats $\mathrm{J} 2: \mathrm{e} 1$

Normile D (2008) The promise and pitfalls of clinical trials overseas. Science 322:214-216

Normile D (2009) Scientists puzzle over Ebola-Reston virus in pigs. Science 323:451

Peeling RW, Mabey D, Fitzgerald DW et al (2004) Avoiding HIV and dying of syphilis. Lancet 364:1561-1563

Peeling RW, Smith PG, Bossuyt PMM (2006) A guide for diagnostic evaluations. Nat Revs Microbiol 4:52-56

Povelones M, Waterhouse RM, Kafatos F et al (2009) Leucine-rich repeat protein complex activates mosquito complement in defense against Plasmodium parasites. Science 324:258-261

Rothwell PM (2006) Funding for practice-oriented clinical research. Lancet 368:262-266

Taubes G (2008) The bacteria fight back. Science 321:356-361

White NJ (2006) Clinical trials in tropical diseases: a politically incorrect view. Trop Med Intern Health 11:1483-1484

World Health Organization (2004) World report on knowledge for better health. WHO, Geneva

World Health Organization (2006) World health statistics 2006. WHO, Geneva

World Health Organization (2008) World health statistics 2008. WHO, Geneva 